\title{
Engineering Graduate Attribute Development (EGAD) Project
}

\author{
Brian Frank \\ Queen's University \\ Susan Fostaty-Young \\ Queen's University \\ Nasser Saleh \\ Queen's University
}

\author{
Susan McCahan \\ University of Toronto \\ Peter Ostafichuck \\ University of British Columbia
}

\author{
K. Christopher Watts \\ Dalhousie University. \\ Peter Wolf \\ University of Guelph
}

$\frac{\text { http://engineering.queensu.ca/egad }}{\text { egad@appsci.queensu.ca }}$

\begin{abstract}
This paper describes the objectives and progress of the Engineering Graduate Attribute Development (EGAD) project. This is a Canadian collaborative project sponsored by the National Council of Deans of Engineering and Applied Science (NCDEAS), in collaboration with Engineers Canada. EGAD's mandate is to develop resources and training for assessing graduating student attributes in undergraduate engineering programs for the purpose of improving the quality of engineering education in Canada, specifically in response to CEAB's graduate attribute requirements.
\end{abstract}

\section{Introduction}

In 2009 the Canadian Engineering Accreditation Board $(\mathrm{CEAB})$ began requiring engineering programs to assess attributes of graduating students. The National Council of Deans of Engineering and Applied Science (NCDEAS) has been working with $\mathrm{CEAB}$ to operationalize the process of graduate attribute assessment. One of the responses was the creation of a project to develop resources and provide assistance to engineering faculty and administrators.

The Engineering Graduate Attribute Development (EGAD) Project is a collaboration of engineering educators and educational developers from across Canada. The approach we are adopting is one of collegial cooperation and one that borrows from international colleagues and from other disciplines engaged in similar transitions to outcomes-based education. It is anticipated that in learning from others' experiences we can work toward assuring positive teaching and learning experiences for students, educators and the institutions across Canada that provide engineering programs.

The challenge of transition is a significant one. The traditional approach to accreditation is to be supplemented by one that attempts to assess the quality of programming by assessing students' learning through specifically identified outcomes. The approach requires continuous program-wide assessment and a much greater degree of faculty and student engagement in the process. The new accreditation focuses on technical expertise as well as on the integration of supporting professional skills, a movement from assessing discrete competencies to one of assessing integrated professional engineering competence through the identification of graduate attributes.

This reflects a broader trend in Canadian higher education, and professional programs specifically, to require programs to create a quality assurance process. For example, this is reflected in the Council of Ontario University's graduate and undergraduate degree level expectations.

While the challenges are significant the opportunities are even greater. If the approach taken to curriculum re-design respects the care, education, development, resources and efforts of engaging in outcomes-based education, the concomitant change in accreditation could transform not only the engineering curriculum, but all those who are engaged in the field of engineering: educators; faculty; students; practitioners; and influence the positive role that engineers can have for the betterment of Canada.

The EGAD working group has adopted an approach to supporting outcomes-based curriculum development 
based on the following 4 tenets of effective curriculum practice.

1. Outcomes-based curriculum development is a process of the continuous improvement of sustainable practices

2. Outcomes achievement is a responsibility shared by faculty and students.

3. There is a culture of autonomy and academic freedom within courses and curriculum in higher education

4. A scholarly approach to curriculum development includes processes that are faculty-driven, data-informed and literaturesupported. The process is further supported by a scholarly approach to analysis, application, teaching and assessment.

Through them our intention is to engage engineering educators, students and the engineering community in ensuring that Schools of Engineering graduate students who are ready to meet the needs of an increasingly changing and complex society, while at the same time supporting the tenets of academic freedom and respecting the learning culture and resource parameters of individual institutions.

\section{Outcomes-based curriculum development is a process of the continuous improvement of sustainable practices}

One of the many positive aspects of outcomes-based accreditation is to position learning within a basic framework of expectations such that the design, delivery and assessment of learning in a program can be understood by faculty and students. To that end, the working group is committed to identifying resources and frameworks of learning that might help schools of engineering operationalize the 12 Graduate Attributes identified by CEAB, support the use of aligned outcomes, assessment and instructional strategies, and that facilitate communication about expectations for students' learning.

Ongoing engagement in the improvement of curriculum happens through the ongoing collection and interpretation of data, critical reflection on the outcomes and on how students' achievement might be furthered in the curriculum. This can take place through a variety of activities that include: curriculum committee meetings and departmental retreats; educator development workshops; opportunities for development in the design and delivery of courses and their connections to the outcomes; and collaborative development of rubrics, portfolios and other means to demonstrate achievement of the outcomes. The EGAD working group is committed to identifying resources for these purposes.

To be sustainable, practices must support students' ongoing learning and be responsive to the everemerging learning needs of soon-to-be professional engineers. Developing sustainable practices for intentional and scholarly approaches to curriculum development must be considered if one of the goals of outcomes-based accreditation is to foster continuous improvement processes for engineering curriculum. A second step toward sustainability is in ensuring that the evaluation process is specific enough that it delineates the level of cognitive and other complexities of the expected learning processes while remaining elastic enough to accommodate and support emerging trends and content demands.

\section{Outcomes achievement is a shared responsibility}

Though schools can provide students with opportunities to develop outcomes, students have a shared responsibility to demonstrate outcomes achieved and to shape their own learning.

Engaging students at the outset of their engineering education and embedding ways for students to integrate and demonstrate the achievement of outcomes across the curriculum through the use of portfolios, capstone experiences and experiential learning will further curriculum initiatives and encourage students to consider their own professional development.

It must also be noted that many attributes develop outside the planned curriculum. Summer and part-time jobs, co-op placements, student politics, clubs, incidental contact with faculty or peers, and other types of co-and extra-curricular activities all contribute to student success and outcomes achievement. It is in every program's and every student's best interest to find ways to document and acknowledge the contributions and effects of such extra- and co-curricular learning.

\section{There is a culture of autonomy and academic freedom within courses and curriculum in higher education}

There is a considerable degree of educator autonomy with respect to course content and delivery strategies in post-secondary institutions. Because we value the 
positive effects of that autonomy on the student learning experience we have adopted what might best be described as a moderate approach to the process of articulation of learning outcomes and in the operationalizing of Graduate Attributes. It has been our experience that highly prescriptive outcomes accompanied by one-to-one corresponding assessment may inadvertently reduce learning to the accumulation of isolated competencies rather than support the development of integrated competence. As well, such highly prescriptive approaches tend to thwart innovation in the curriculum over time. Over-explicit outcomes and assessment directly linked to a specific outcome for accreditation purpose may impose unintended limitations on learning.

\section{Curriculum development is a scholarly activity}

A scholarly approach to curriculum development includes processes that are faculty-driven, datainformed and literature-supported. The process is further supported by a scholarly approach to analysis, application, teaching and assessment.

The hope is that a more scholarly approach to engineering education will emerge, as it has in other countries, by involving dissemination of results and peer review.

As has been established in the US and in other disciplines in Canada (e.g. health sciences), communities of practice across institutions, conferences, workshops and informal networking opportunities can be developed to support this work.

\section{Recent activities}

Since the group's formation EGAD's activities have been concentrated on:

1. Creation of philosophical approach

2. The development of a resource-based website

3. Dissemination of information and processes through workshops and presentations

4. Communication with $\mathrm{CEAB}$ and engineering programs

The website, released in early 2011, can be accessed at http://engineering.queensu.ca/egad. It includes:

- PowerPoint presentations used for workshops on graduate attribute assessment and curriculum mapping
- a glossary of terms specific to the 2010 Questionnaire for Evaluation of an Engineering Program (Criteria 3.1)

- Pertinent research-based literature

- Information on learning assessment

- Suggestions on curriculum mapping and continuous curriculum review

- Sample case approaches to data informed curriculum review

- Contact information and invitations to contact EGAD members for resources and support

The website includes case studies and material developed former and current EGAD member programs, including UBC, U Calgary, Guelph, Toronto, Queen's, and Dalhousie.

\section{Upcoming resources and activities}

The current thrust is the development of training modules appropriate to faculty, administration, and CEAB site visitors. Lecture-captured version of a training workshop are also planned, and real-time and archived webinars.

Over the coming year we hope to establish mentoring/support relationships between recently accredited institutions and institutions coming up for accreditation, and a repository of best practices, resources and tools solicited from institutions willing to share their experience.

The University of Guelph has developed a web-based curriculum mapping tool known as CurriKit. EGAD is partnering with that team to investigate making that more broadly available to programs.

\section{Conclusions}

The EGAD group looks forward to collaborating with faculty and students across the country to share ideas, processes, and resources. As programs go through the process of accreditation and a complete cycle of data collection, analysis, and program change, more resources will become available. 